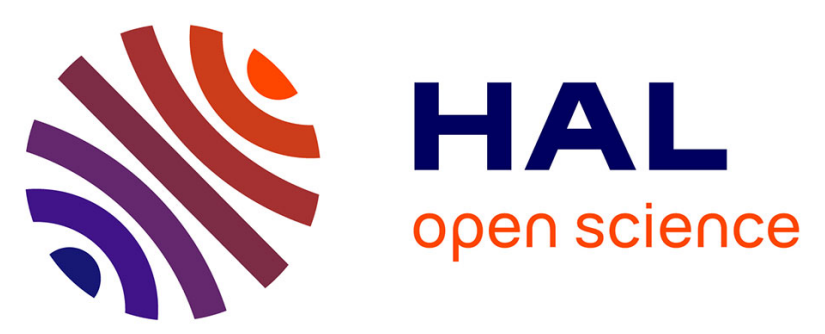

\title{
Modulation of beta power in EEG during discrete and continuous motor imageries
}

\author{
Sébastien Rimbert, Cecilia Lindig-León, Mariia Fedotenkova, Laurent \\ Bougrain
}

\section{- To cite this version: \\ Sébastien Rimbert, Cecilia Lindig-León, Mariia Fedotenkova, Laurent Bougrain. Modulation of beta power in EEG during discrete and continuous motor imageries. 8th International IEEE EMBS Con- ference On Neural Engineering, May 2017, Shanghai, China. hal-01484503}

\section{HAL Id: hal-01484503 \\ https://hal.science/hal-01484503}

Submitted on 7 Mar 2017

HAL is a multi-disciplinary open access archive for the deposit and dissemination of scientific research documents, whether they are published or not. The documents may come from teaching and research institutions in France or abroad, or from public or private research centers.
L'archive ouverte pluridisciplinaire HAL, est destinée au dépôt et à la diffusion de documents scientifiques de niveau recherche, publiés ou non, émanant des établissements d'enseignement et de recherche français ou étrangers, des laboratoires publics ou privés. 


\title{
Modulation of beta power in EEG during discrete and continuous motor imageries
}

\author{
Sébastien Rimbert ${ }^{1,2,3}$, Cecilia Lindig-León ${ }^{1,2,3}$, Mariia Fedotenkova ${ }^{1,2,3}$ and Laurent Bougrain ${ }^{2,1,3}$
}

\begin{abstract}
In most Brain-Computer Interfaces (BCI) experimental paradigms based on Motor Imageries (MI), subjects perform continuous motor imagery (CMI), i.e. a repetitive and prolonged intention of movement, for a few seconds. To improve efficiency such as detecting faster a motor imagery, the purpose of this study is to show the difference between a discrete motor imagery (DMI), i.e. a single short MI, and a CMI. The results of experiment involving 13 healthy subjects suggest that a DMI generates a robust post-MI event-related synchronization (ERS). Moreover event-related desynchronization (ERD) produced by DMI seems less variable in certain cases compared to a CMI.
\end{abstract}

\section{INTRODUCTION}

Motor imagery (MI) is the ability to imagine performing a movement without executing it[1]. MI has two different components, namely the visual-motor imagery and the kinesthetic motor imagery (KMI) [2]. KMI generates an event-related desynchronization (ERD) and an event-relatedsynchronization (ERS) in the contralateral sensorimotor area, which is similar to the one observed during the preparation of a real movement (RM) [3]. Compared to a resting state, before a motor imagery, firstly there is a gradual decrease of power in the beta band $(15-30 \mathrm{~Hz})$ of the electroencephalographic signal, called ERD. Secondly, a minimal power level is maintained during the movement. Finally, from 300 to 500 milliseconds after the end of the motor imagery, there is an increase of power called ERS or post-movement beta rebound with a duration of about one second.

Emergence of ERD and ERS patterns during and after a MI has been intensively studied in the Brain-Computer Interface (BCI) domain [4] in order to define detectable commands for the system. Hence, a better understanding of these processes could allow for the design to better interfaces between the brain and a computer system. Additionally, they could also play a major role where MI are involved such as rehabilitation for stroke patients [5] or monitoring consciousness during general anesthesia [6].

Currently, most of the paradigms based on MIs require the subject to perform the imagined movement several times for a predefined duration. In this study, such a task is commonly referred to as a continuous motor imagery (CMI). However, first the duration of the experiment is long, second a succession of flexions and extensions generates an overlapping of ERD and ERS patterns making the signal less detectable.

\footnotetext{
*This work has been supported by the Inria project BCI LIFT

${ }^{1}$ Neurosys team, Inria, Villers-lès-Nancy, F-54600, France

${ }^{2}$ Artificial Intelligence and Complex Systems, Université de Lorraine, LORIA, UMR 7503, Vandœuvre-lès-Nancy, F-54506

${ }^{3}$ Neurosys team CNRS, LORIA, UMR 7503, Vandœuvre-lès-Nancy, F54506
}

In fact, one simple short MI, referred in this article as a discrete motor imagery (DMI), could be more useful for two reasons. Firstly, a DMI could be used to combat fatigue and boredom for BCI-users improving ERD and ERS production [7]. Secondly, the ERD and ERS generated by the DMI could be detectable at a higher quality and more rapidly compared to a CMI. This was found in a previous study that established a relationship between the duration of MI and the quality of the ERS extracted and showed that a brief MI (i.e. 2 seconds MI) could be more efficient then a sustained MI [8]. Our main hypothesis is that a DMI generates robust ERD and ERS patterns which could be detectable by a BCIsystem. To analyze and compare the modulation of beta band activity during a RM, a DMI and a CMI, we computed timefrequency maps, the topographic maps and ERD/ERS\%.

\section{MATERIAL AND METHODS}

\section{A. Participants}

13 right-handed healthy volunteer subjects took part in this experiment ( 7 men and 6 women, from 19 to 43 years old). They had no medical history which could have influenced the task. All subjects gave their agreement and signed an information consent form approved by the ethical INRIA committee before participating.

1) Real movement: The first task consisted of an isometric flexion of the right index finger on a computer mouse. A low frequency beep indicated when the subject had to execute the task.

2) Discrete imagined movement: The second task was a DMI of the previous real movement.

3) Continuous imagined movement: The third task was a CMI during four seconds of the real movement of the first task. More precisely, the subject imagined several (around four) flexions and extensions of the right index finger. This way, the DMI differed from the CMI by the repetition of the imagined movement. The number of imagined flexions was fixed (4 MIs). For this task, two beeps, respectively with low and high frequencies, separated by a four second delay, indicated the beginning and the end of the CMI.

\section{B. Protocol}

Each of the three tasks introduced in section II corresponds to a session. The subjects completed three sessions during the same day. All sessions were split into several runs. Breaks of a few minutes were planned between sessions and between runs to avoid fatigue. At the beginning of each run, the subject was told to relax for 30 seconds. Condition 1 corresponded to RMs was split into 2 runs of 50 trials. 
Conditions 2 and 3 corresponded to discrete and continuous imagined movements, respectively, was split into 4 runs of 25 trials. Thus, 100 trials were performed by subjects for each task. Each experiment began with condition 1 as session 1. Conditions 2 and 3 were randomized to avoid possible bias cause by fatigue, gel drying or another confounding factor.For conditions 1 and 2, the timing scheme of a trial was the same: one low frequency beep indicated the start followed by a rest period of 12 seconds. For condition 3, a low frequency beep indicated the start of the MI to do during 4 seconds, followed by a rest period of 8 seconds. The end of the MI is announced by a high frequency beep (Fig. 1).

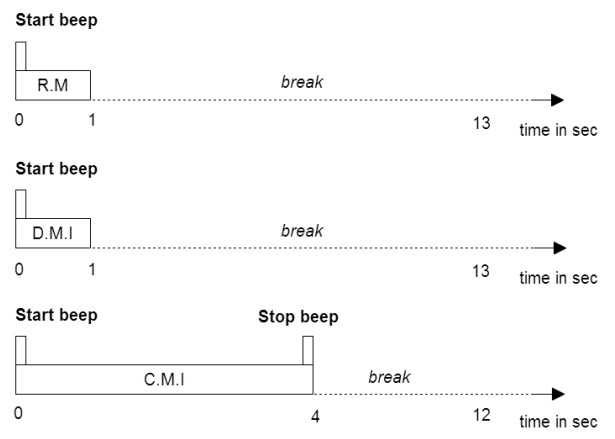

Fig. 1. Timing schemes of a trial for each task: Real Movement (RM, top); Discrete Motor Imagery (DMI, middle); Continuous Motor Imagery (CMI, bottom). The DMI and CMI sessions are randomized.

\section{Electrophysiological data}

EEG signals were recorded through the OpenViBE [9] platform with a commercial REFA amplifier developed by TMS International. The EEG cap was fitted with 9 passive electrodes re-referenced with respect to the common average reference across all channels over the extended international 10-20 system positions. The selected electrodes are FC3, C3, CP3, FCz, Fz, CPz, FC4, C4, CP4. Skin-electrode impedances were kept below $5 \mathrm{k} \Omega$.

\section{EEG data analysis}

We performed time-frequency analysis using spectrogram method(Fig. 2). The spectrogram is a squared magnitude of the short-time Fourier transform. As the analysis window in the method of spectrogram we used Gaussian window with $\alpha=2.5$ [10] with overlap by one time point between the subsequent segments. The length of the window was chosen such as to give the frequency resolution $\Delta f=1 \mathrm{~Hz}$.

To evaluate more precisely this modulation we computed the ERD/ERS\% using the "band power method" [3] with a matlab code. First, the EEG signal is filtered between 15-30 $\mathrm{Hz}$ (beta band) for all subjects using a 4th-order Butterworth band-pass filter. Then, the signal is squared for each trial and averaged over trials. Then it is smoothed using a 250millisecond sliding window with a $100 \mathrm{~ms}$ shifting step. Finally, the averaged power computed for each window was subtracted and then divided by the averaged power of a baseline corresponding to 2 seconds before each trial.
In addition, we computed the topographic maps of the ERD/ERS\% modulations for all subjects (see Fig. 3).

\section{RESULTS}

\section{A. Electrophysiological results}

To verify if a DMI generates ERD and ERS patterns which could be detectable by a CMI, we studied the following three features: (i) the time-frequency analysis for the electrode $\mathrm{C} 3$, (ii) the relative beta power for the electrode $\mathrm{C} 3$ and (iii) the topographic map built from the 9 selected electrodes. Electrode C3 is suitable for monitoring right hand motor activity. A grand average was calculated over the 13 subjects. We used a Friedman's test to analyze whether ERS were significantly and respectively different during the three conditions. Because participants were asked to close her eyes, the alpha band was disturbed (confirmed by the time-frequency analysis) and not considered for this study. Consequently values corresponding to the desynchronization appears smaller because they were only analyzed in the beta band. For this reason, section III is mainly focused on the ERS.

1) Real movement: Fig. 2.A illustrates a strong synchronization in the $17-20 \mathrm{~Hz}$ band appearing 2 seconds after the start beep and confirmed the activty in the beta band. The ERD/ERS\% averages (Fig. 2.D) indicate that one second after the cue, the power in the beta band increases by around $80 \%$, reaches its maximum and returns to the baseline 4 seconds after. The evolution from ERD to ERS is rapid (less than one second) and should be linked to the type of movement realized by the subjects. Interestingly, each subject (except Subject 13) has a same ERD/ERS\% profile (i.e. a strong beta rebound) after the real movement. Subject 13 has no beta rebound after the movement but has a stronger ERD, it is particularly true for the other conditions. The grand average topographic map (Fig. 3) shows that the ERS is more important on the area of the electrode C3. However, the ERS is also present around other electrodes, as well as the ipsilateral one.

2) Discrete motor imagery: Fig. 2.B shows a strong modulation in the $16-22 \mathrm{~Hz}$ band starting 2 seconds after the start beep. The ERS post-MI reaches $28 \%$ which is less stronger compare to the other tasks (Fig. 2.E). Some subjects (S1, S2, S5, S6, S10) have a stronger robust ERS produced by DMI while others have no beta rebound. This confirms that a DMI could be used in BCI domain. The lack of beta rebound (S3, S4, S11) could be caused to the difficulty of the DMI task. Indeed, post-experiment questionnaires showed that some subjects had difficulties in performing this task. The grand average (Fig. 3) shows desynchronization around $5 \%$ over the $\mathrm{C} 3$ area. One second later, the beta rebound appears, and is more present around the C3 area.

3) Continuous motor imagery: During the CMI, the subjects imagined several movements in a time window of $4 \mathrm{sec}$ onds. Fig. 2.C show a global decrease of activity during the CMI and stronger modulation in $16-21 \mathrm{~Hz}$ after the MI. The results of the grand average showed a low desynchronization during this time window. It is interesting to note that some 
A
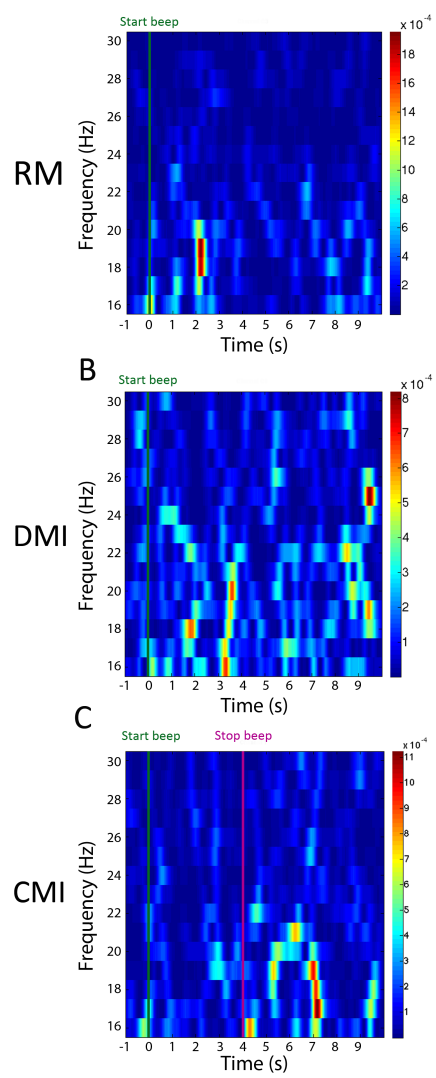

D
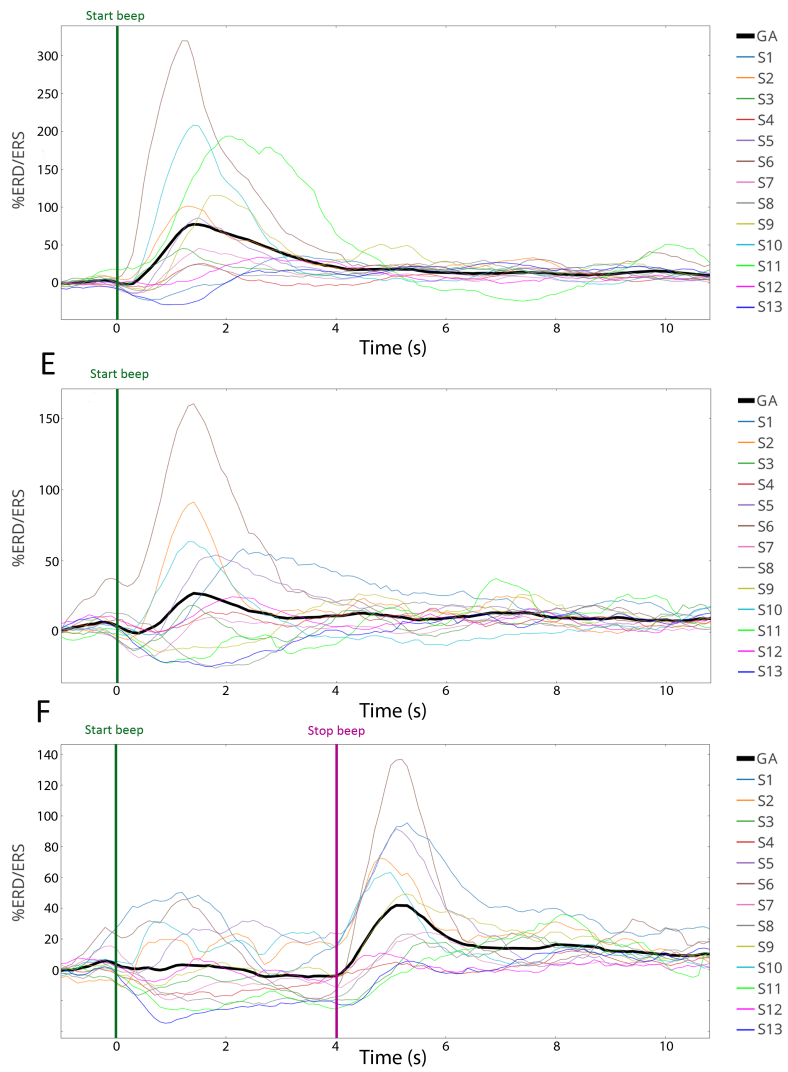

Fig. 2. Left side: time-frequency grand average $(\mathrm{n}=13)$ analysis for the RM (A), the DMI (B), the CMI (C) for electrode $C_{3}$. A red color corresponds to strong modulations in the band of interest. Right side: grand average ERD/ERS\% curves (in black, GA) estimated for the RM (D), the DMI (E), the CMI (F) within the beta band $(15-30 \mathrm{~Hz})$ for electrode $C_{3}$. The average for each subject is also presented.

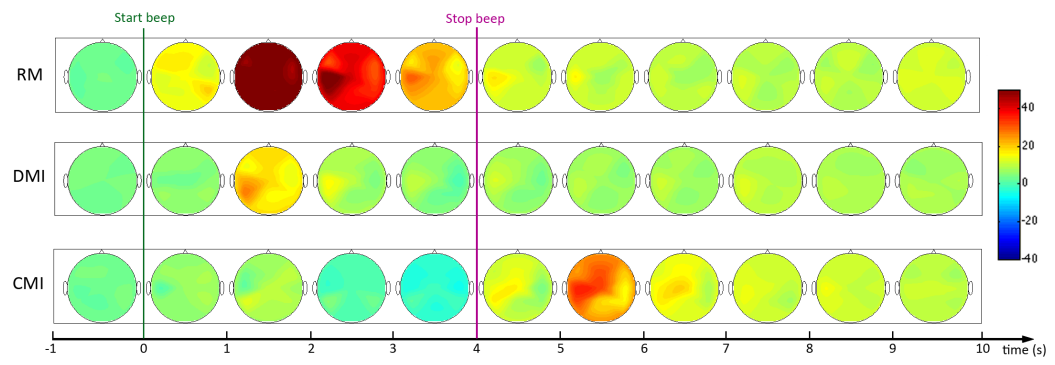

Fig. 3. Topographic map of ERD/ERS\% (grand average, $\mathrm{n}=13$ ) in the $15-30 \mathrm{~Hz}$ beta band during Real Movement (top), Discrete Motor Imagery (middle) and Continuous Motor Imagery (bottom). The red color corresponds to a strong ERS (+50\%) and a blue one to a strong ERD ( $-40 \%$ ). The green line indicates when the start beep sounds and the purple line indicates when the end beep sounds to stop the CMI. On this extrapolated map only recorded electrode will be considered (FC3, C3, CP3, FCz, Fz, CPz, FC4, C4, CP4).

subjects (S2, S10) have no desynchronization during the CMI task and could have a negative effect on the classification phase. Other subjects (S6, S1, S7) have a different profile which shows that a first ERS is reached one second after the beginning of the CMI, then the power increases and decreases again, being modulated during 3 seconds. Indeed, this ERD can be considered as the concatenation of several ERDs and ERSs due to the realization of several MIs. Indeed, for some subjects (S1, S6 or S9) the first ERD (23\%) is reached during the first second after the MI. The topographic map shows that during the first second after the start beep, an ERD is lightly visible, but there is difficulty to identify a synchronization or a desynchronization. Understanding of individual ERD and ERS profiles between subjects for the CMI task is crucial to improve the classification phase in a BCI.

4) Comparison between RM, DMI and CMI: We observe that the ERS is stronger for a real movement. In fact, the beta rebound is $60 \%$ larger for a RM than for a MI. Although the ERS is stronger during a DMI than a CMI for some subjects (S2 and S6), this result is not statistically significant according to the Friedman test. The ERS of the CMI is 
stronger than the ERS of a DMI in average. For both DMI and CMI, the ERD is stronger and lasts longer than for the real movement. For some subjects (S1, S6 and S10) ERD produced during the CMI is more variable and seems to be the result of a succession of ERD and ERS generated by several MI.

\section{DISCUSSION}

The subjects carried out voluntary movements, DMI and CMI of an isometric flexion of the right hand index finger. Results show that the power of the beta rhythm is modulated during the three tasks. The comparison between ERSs suggests that subjects on average have a stronger ERS during a CMI than a DMI. However, this is not the case for all subjects.

\section{A. EEG system}

It is well established that a large number of electrodes allows to have a good estimation of the global average potential of the whole head [11]. Although we are focused on specific electrodes, our results were similar by using method of the derivation, which corresponded to the literature. We choosed to study C3 without derivation because we are interested to designing a mnimal system to detect ERD and ERS during general anesthesia conditions.

\section{B. ERD/ERS modulation during real movements}

The results are coherent with previous studies describing ERD/ERS\% modulations during motor actions. The weakness of the ERD can be linked to the instruction that was focused more on the precision than the speed of the movement [12].

\section{ERS modulation during motor imageries}

The results show that the beta rebound is lower after a DMI or a CMI than after a real movement, which has been already been demonstrated previously[13]. However, the novelty is the beta rebound is stronger on average after a CMI than DMI for a few subjects.

\section{ERD modulation during continuous motor imagery}

When the subjects performed the CMI, the ERD was highly variable during the first 4 seconds. For some subjects, our hypothesis is there are some intern-ERD and internERS into this period. The difficulty is that the CMI involve several MI, that are not synchronized across trials, unlike the DMI which starts and ends at roughly the same time for each trial, due to the cue. Normally, for continuous real movement, the ERD was sustained during the execution of this movement[14]. However, in our data it is possible to detect several ERDs during the 4 seconds of CMI where the subject performed 3 or 4 MIs. This assumes that the ERD and ERS components overlap in time when we perform a CMI. Several studies already illustrate the concept of overlap of various functional processes constituting the beta components during RMs [15]. This could explain why the ERD during a CMI could be less detectable and more varied than the ERD during a DMI. To validate this hypothesis, we plan to design a new study to explore how two fastsuccessive movements (or MIs) can affect the signal in the beta frequency band.

\section{CONCLUSIONS}

This article examined the modulation of beta power in EEG during a real movement, a discrete motor imagery (DMI) and a continuous motor imagery (CMI). We showed that during a real voluntary movement corresponding to an isometric flexion of the right hand index finger a low ERD appeared, and was followed by a rapid and powerful ERS. Subsequently, we showed that the ERD and ERS components were still modulated by both a DMI and a CMI. The ERS is present in both cases and shows that a DMI could be used in BCI domain. In future work, a classification based on the beta rebound of a DMI and a CMI will be done to complete this study and confirm future impact of DMI task in BCIdomain to save time and avoid fatigue.

\section{REFERENCES}

[1] L. Avanzino, A. Giannini, A. Tacchino, E. Pelosin, P. Ruggeri, and M. Bove, "Motor imagery influences the execution of repetitive finger opposition movements," Neuroscience Letters, vol. 466, no. 1, pp. 11 $-15,2009$

[2] C. Neuper, R. Scherer, M. Reiner, and G. Pfurtscheller, "Imagery of motor actions: Differential effects of kinesthetic and visual-motor mode of imagery in single-trial $\{\mathrm{EEG}\}$, , Cognitive Brain Research, vol. 25 , no. 3 , pp. $668-677,2005$.

[3] G. Pfurtscheller and F. H. Lopes da Silva, "Event-related eeg/meg synchronization and desynchronization: basic principles," Clin Neurophysiol, vol. 110, no. 11, pp. 1842-57, Nov 1999.

[4] E. W. W. Jonathan Wolpaw, Ed., Brain-Computer Interfaces: Principles and Practice. Oxford university press, 2012.

[5] A. J. Butler and S. J. Page, "Mental practice with motor imagery: evidence for motor recovery and cortical reorganization after stroke." Arch Phys Med Rehabil., vol. 87, pp. S2-11, dec. 2006.

[6] Y. Blokland, J. Farquhar, J. Lerou, J. Mourisse, G. J. Scheffer, G.-J. van Geffen, L. Spyrou, and J. Bruhn, "Decoding motor responses from the eeg during altered states of consciousness induced by propofol," Journal of Neural Engineering, vol. 13, no. 2, p. 026014, 2016.

[7] M. Ahn and S. C. Jun, "Performance variation in motor imagery braincomputer interface: a brief review." J Neurosci Methods, vol. 243, pp. 103-110, Mar 2015.

[8] E. Thomas, J. Fruitet, and M. Clerc, "Investigating brief motor imagery for an erd/ers based bci." Conf Proc IEEE Eng Med Biol Soc, vol. 2012, pp. 2929-2932, 2012.

[9] Y. Renard, F. Lotte, G. Gibert, M. Congedo, E. Maby, V. Delannoy, O. Bertrand, and A. Lécuyer, "Openvibe: An open-source software platform to design, test and use brain-computer interfaces in real and virtual environments," Presence : teleoperators and virtual environments, vol. 10, no. 1, 2010.

[10] F. J. Harris, "On the use of windows for harmonic analysis with the discrete Fourier transform," Proceedings of the IEEE, vol. 66, no. 1, pp. 51-83, 1978.

[11] J.Dien, "Issues in the application of the average reference: review, critiques, and recommendations," Behavior Research Methods, vol. 30, p. 34, 1998.

[12] B. Pastötter, F. Berchtold, and K.-H. T. Bäuml, "Oscillatory correlates of controlled speed-accuracy tradeoff in a response-conflict task," Hum Brain Mapp, vol. 33, no. 8, pp. 1834-49, Aug 2012.

[13] A. Schnitzler, S. Salenius, R. Salmelin, V. Jousmaki, and R. Hari, "Involvement of primary motor cortex in motor imagery: a neuromagnetic study." Neuroimage, vol. 6, no. 3, pp. 201-208, Oct 1997.

[14] N. Erbil and P. Ungan, "Changes in the alpha and beta amplitudes of the central eeg during the onset, continuation, and offset of longduration repetitive hand movements." Brain Res, vol. 1169, pp. 44-56, Sep 2007.

[15] B. E. Kilavik, M. Zaepffel, A. Brovelli, W. A. MacKay, and A. Riehle, "The ups and downs of beta oscillations in sensorimotor cortex." Exp Neurol, vol. 245, pp. 15-26, Jul 2013. 